\title{
Radio Behavior of Solar Post-Flare Loops
}

\author{
M. Wang ${ }^{1}$, R. X. Xie ${ }^{1}$, H. D. Chen ${ }^{1}$, and Y. H. Yan ${ }^{2}$ \\ ${ }^{1}$ Yunnan Observatory, National Astronomical Observatories, CAS, Kunming 650011, China \\ ${ }^{2}$ National Astronomical Observatories, CAS, Beijing 100012, China
}

\begin{abstract}
The spectral and source characteristics of a complex radio burst observed with the spectrometers of China and the Nobeyama Radio Heliograph are analyzed. This burst presents two separate burst peaks occurred in different frequency range (broad-band microwave and narrow-band decimeter wavelengths). We stress that the late phase radio bursts in decimeter wavelength corresponding to the post-flare loops may be the radio homologous flare.
\end{abstract}

Keywords. Sun: radio radiation

\section{Observations and Data Processing}

Figure 1 shows the time profiles of the bursts on 19 Oct. 2001 observed with the spectrometers $(0.65-7.6 \mathrm{GHz})$ of China. From these profiles of radio bursts, we can clearly distinguish two group peaks with a time interval (peak - peak) of about 25 minutes. Therefore, these radio bursts may be believed the homology-like radio flares.

Figure 5 shows the evolution of the radio sources (NoRH, $17 \mathrm{GHz}$ ). Figures $5 \mathrm{a}$ and $5 \mathrm{~b}$ show the preexisting magnetic unipolar (positive V) and a gradual radio sources superimposed on the unipolar (marked by 'A') before the beginning of radio burst. Figure 2c shows a newly emerging magnetic polar (negative V, marked by 'B') and a another radio source above the newly emerging polar, at the beginning of radio burst. Two opposite polarities may be reconnect to form an inverted 'Y-type' structure leading to the tworibbon flare related to the broad-band radio burst; Figure 5d shows another two newly emerging magnetic polarity (positive $\mathrm{V}$, marked by 'C' and ' $\mathrm{D}$ '). This time have appeared quadrupolar structures; Figure 5e compared with Figure 5c, disappear just newly emerging two positive polar. However, the positive polar $\mathrm{C}$ has changed into negative polar $\mathrm{E}$; Figure 51 shows the finally opposite two polar. It would be this magnetic configuration resulting in the late phase decimetric burst occurred near the apex of post-flare loop.

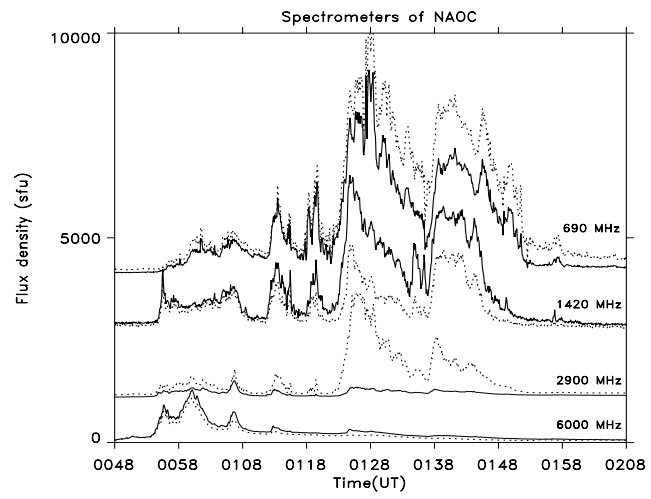

Figure 1. The spectrogram of radio bursts on 19 Oct., 2001. The dashed and solid lines represent the right and left polarization, respectively. 


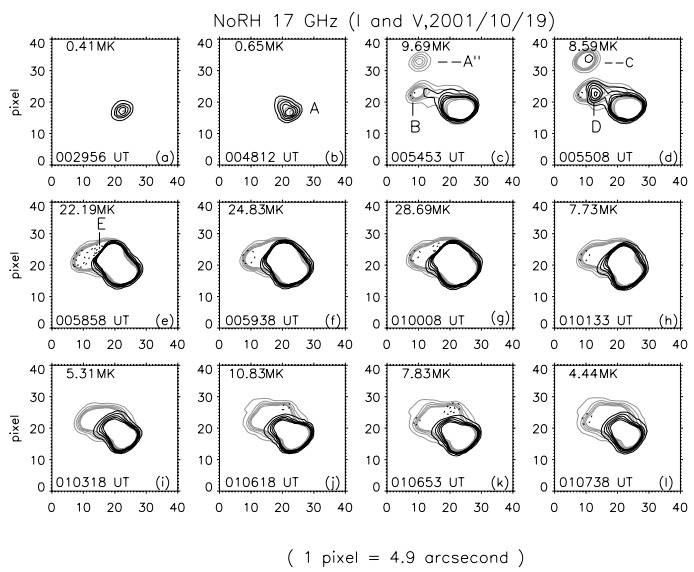

Figure 2. The evolution of NoRH radio sources at $17 \mathrm{GHz}$. The thin lines present the Stocks $\mathrm{I}$, the thick solid and dashed lines present Stocks $\mathrm{V}^{+}$and $\mathrm{V}-$, respectively.

\section{Analysis}

According to Takakura and Scalise (1970) and the frequency range of decimitric burst we get the expanding loops heights of $20 \mathrm{Mm}$. In the impulsive phase of two-ribbon flare the magnetic field lines are dragged upward by the rise filament. The cusp point of an inverted 'Y-type' magnetic configuration slowly rise when successive field lines closes down. The broadband radio burst seems to present the energy release sites which are near the low height, while the narrow band decimetric bursts is near the top of post-flare loops. Therefore magnetic configurations must be variant for applying to the later bursts during the flare. We should consider the existence of a class of bipolar flare model termed an inverted 'Y-type' structure (Aschwanden, 2002). The anti-parallel magnetic fields are supposed to be created the rising Y-type reconnection point that explains the increasing height of the post-flare loops with time (Masuda et al., 1995).

\section{Summary}

The main finding is: the single-loop (bipolar) configuration produced by the newly emerging flux causes a double-ribbon flare (corresponding to broad-band microwave burst), and later homologous flare (corresponding to narrow-band decimetric burst). Our observations support the models of 'Y-type' with bipolar and 'multi-loop' with tripolar or quadrupolar structures. This model involves a dissipative process that opens the magnetic field of an active region. This process allow the field to re-form and to release energy into the cusped arcade structure. It is consistent with a quasi-open inverted 'Y-type' configuration (Aschwanden, 2002).

\section{Acknowledgements}

This research is supported by the National NSF of China grant (No. 10333030, and 10473020), and the foundation of the Chinese Academy of Sciences.

\section{References}

Aschwanden, M. J. 2002, Space Science Reviews, 101/1-2, 1

Masuda, S., Kosugi, T., Hara, H., Sakao, T., Shibata, K., and Tsuneta, S. 1995, Publ. Astron. Soc. Japan, 47, 677

Takakura, T. and Scalise, E. Jr. 1970, Solar Physics, 11, 434 